\title{
A New Acquisition Algorithm with Elimination Side Peak for All BOC Signals
}

\author{
Fang Liu and Yongxin Feng \\ School of Information Science and Engineering, Shenyang Ligong University, Shenyang 110159, China \\ Correspondence should be addressed to Yongxin Feng; onceowned_1019@163.com
}

Received 21 January 2015; Accepted 15 March 2015

Academic Editor: Francesco Tornabene

Copyright (C) 2015 F. Liu and Y. Feng. This is an open access article distributed under the Creative Commons Attribution License, which permits unrestricted use, distribution, and reproduction in any medium, provided the original work is properly cited.

\begin{abstract}
A new inhibition side peak acquisition (ISPA) algorithm is proposed for binary offset carrier (BOC) modulated signals, which will be utilized in global navigation satellite systems (GNSS). We eliminate all side peaks of the BOC correlation function (CF) by structuring special sequences composed of PRN code and cycle rectangular sequences. The new algorithm can be applied to both generic sine- and cosine-phased BOC signals, as well as to all modulation orders. Theoretical and simulation results demonstrate that the new algorithm can completely eliminate the ambiguity threat in the acquisition process, and it can adapt to lower SNR. In addition, this algorithm is better than the traditional algorithms in acquisition performance and inhibition side peak ability.
\end{abstract}

\section{Introduction}

With the development and application of global navigation satellite systems (GNSS) [1], GNSS signal receiving methods have become highly valued. Because the acquisition technology is the core of receiving; therefore, it also becomes a focus problem. Thus, mass acquisition algorithms $[2,3]$ are proposed for GNSS signals to improve receiving performance. However, modern GNSS has provided new signals with longer PRN (pseudo random noise) codes and newer modulation methods, which aim to improve the positioning performance. Binary offset carrier (BOC) [4] modulated signals are the most widely used signal families in GNSS, and their side peak characteristics also require the highest technique complexity from GNSS receivers.

BOC modulation signal acquisition techniques focus on recovering the main correlation peak or eliminating ambiguities in the form of side peaks. At present, various techniques are proposed for side peak cancellation and are built on the basis of the correlation function (CF) of the BOC signals. Thus, the side band processing method originated from BPSK-like method $[5,6]$, and then some improved methods [7-9] are proposed. The partial band is obtained by filtering or frequency domain processing in these kind methods, and then the main peak was estimated using similar BPSK characteristic. These kind methods can reduce the influence of subcarrier, but the energy and the necessary information are lost. Thus, the auxiliary signal methods $[10,11]$ are mainly through the local auxiliary signal establishment to reach the purpose of removing side peaks. These kind methods can remove the side peak, but they lack universality. Thus, some effective methods [12-14] are proposed to improve the processing performance. However, these techniques apply only to sine-phased BOC signals. Thus, in [15], a mitigating ambiguity acquisition method is proposed. This technique can counterbalance the undesired side peaks, but it applies only to cosine-phased BOC signals.

In this paper, considering filter restriction and generic deficiency problems in traditional algorithms, we propose an inhibition side peak acquisition algorithm, which is applicable to all orders and to both generic sine- and cosine-phased BOC signals.

\section{BOC Modulation Signal and Acquisition Analysis}

2.1. BOC Modulation Signal. BOC modulation signal is obtained by the product of PRN code and the square wave. The complex form of the BOC signal is expressed as

$$
S(t)=e^{-i \theta} \sum_{k} a_{k} \mu_{\varepsilon T_{s}}\left(t-k \cdot \varepsilon T_{s}-t_{0}\right) C_{T_{s}}\left(t-t_{0}\right),
$$




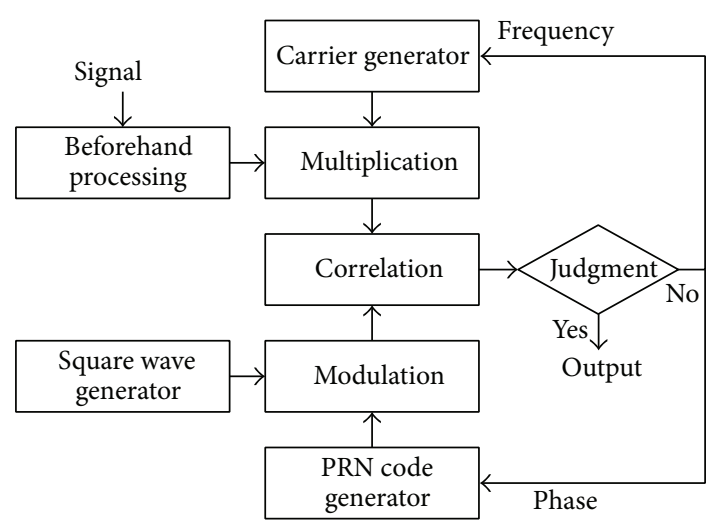

FIGURE 1: The full band acquisition algorithm principle.

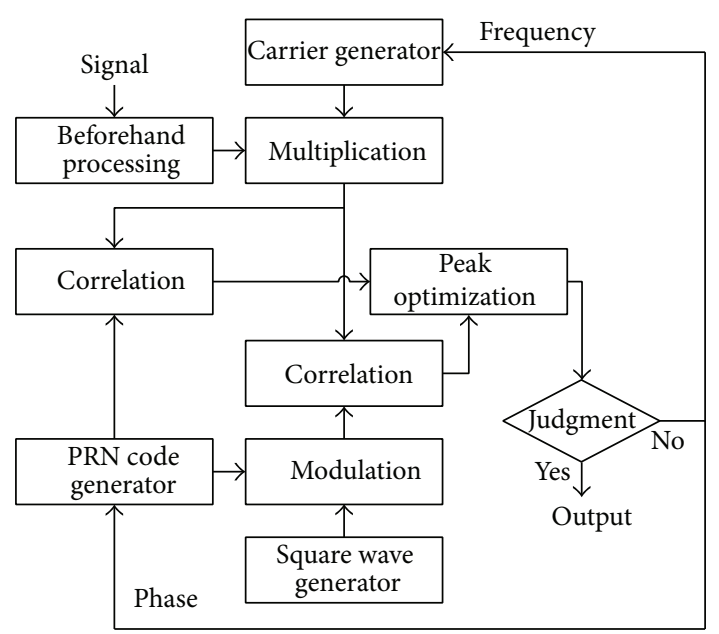

FIGURE 2: The peak optimization acquisition algorithm principle.

where $a_{k}$ is the modulated PRN code, $C_{T_{s}}(t)$ is the subcarrier, $2 T_{s}$ is the subcarrier cycle, $\mu_{n T_{s}}(t)$ is the spread spectrum symbol, $\varepsilon$ is the modulation order, and $\theta$ and $t_{0}$, respectively, express the phase and time offset.

The BOC signal is usually expressed as $\operatorname{BOC}\left(f_{s}, f_{c}\right)$, the frequency of the subcarrier is $f_{s}$ times the benchmark frequency, and the frequency of the PRN code is $f_{c}$ times the benchmark frequency. The benchmark frequency is $1.023 \mathrm{MHz}$. The autocorrelation function of the BOC signal has multiple peaks and passes through zero many times. Its autocorrelation function consists of the positive peaks and the negative peaks, and the number of peaks is $2 \varepsilon-1$. The distance between peaks is $T_{s}$, and each peak height is $(-1)^{l}(\varepsilon-$ $|l|) / \varepsilon$, where $l$ is the serial number of the peaks.

2.2. The Acquisition Analysis. From the perspective of algorithm generality, the acquisition algorithm for BOC modulation signal is usually divided into three categories, namely, the full band acquisition (FBA) algorithm [16], the peak optimization acquisition (POA) algorithm [17], and the single peak recovery acquisition (SPRA) algorithm [18]. Their principles are shown in Figures 1, 2, and 3, respectively.

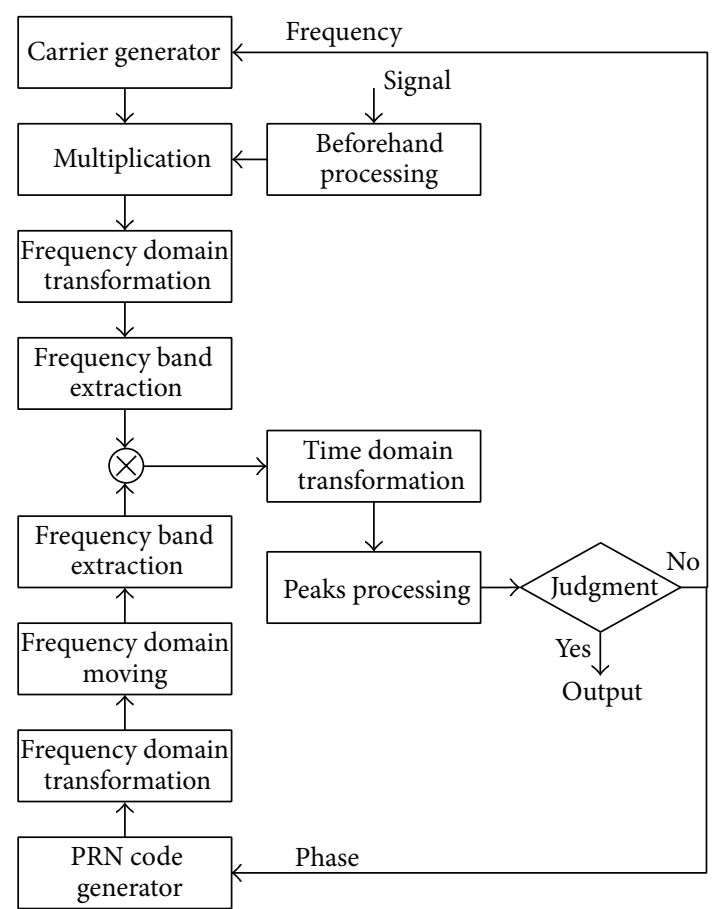

FIGURE 3: The single peak recovery acquisition algorithm principle.

FBA is a class of traditional algorithms, in which the correlation arithmetic is executed between the received signal and the original PRN code modulated by a square wave. POA is a class of improved algorithms, in which multiple correlations are executed to improve the main peak. SPRA is a class of new methods, in which a partial signal is separated from the received signal by the corresponding operations to inhibit the square wave.

\section{ISPA Algorithm Structure}

Let $f_{t}$ be the sampling frequency of the BOC signal, and the frequency of the subcarrier and PRN code are $f_{s}$ times and $f_{c}$ times the benchmark frequency, respectively. Considering square wave modulation characteristics, the product model of the spread spectrum sequence and a series of rectangular sequences is structured, which can be approximately expressed as the BOC base-band signal model. Hence, the base-band signal may be represented by the following equation:

$$
\begin{aligned}
S_{\mathrm{BOC}}(n)= & d(n) C(n) \\
& \cdot \sum_{j=1}^{\varepsilon M}\left((-1)^{j+1} R_{N}(n+N-j N)\right) S_{\Delta}(n)+\lambda_{0}(n),
\end{aligned}
$$

where $d(n)$ is the message, $C(n)$ is the PRN code, $\lambda_{0}(n)$ is the mixed noise function caused by the discarded samples, $S_{\Delta}(n)$ is the frequency error function cause by the front processing, $n$ is the sequence position, $M$ is the number of chips in accumulation time, and $\varepsilon$ is both the modulation order and 


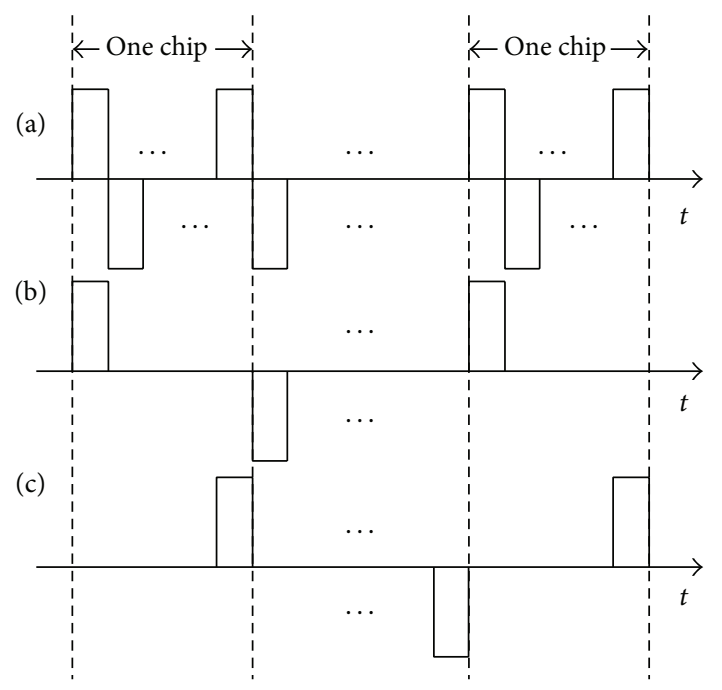

FIGURE 4: The structured process of the rectangular sequence model.

the number of rectangular sequences in one chip, which is expressed as (3). $N$ is both the number of sampling points and the rectangular sequence width, which is expressed as (4). $R_{N}(n+N-j N)$ is a shifting rectangular sequence, which is expressed in (5), where $u(n)$ is the step sequence:

$$
\begin{gathered}
\varepsilon=\frac{2 f_{s}}{f_{c}}, \\
N=\frac{f_{t}}{2 f_{s}}, \\
R_{N}(n+N-j N)=u(n+N-j N)-u(n-j N) .
\end{gathered}
$$

Considering the represented model of the BOC baseband signal, the local rectangular sequence model is structured to inhibit the acquisition of side peaks. The structured process is shown in Figure 4, in which $\varepsilon$ is an odd number. The square wave sequence is shown in Figure 4(a), and the two structured cycle rectangular sequences are shown in Figures 4(b) and 4(c). The cycle rectangular sequences can also be structured for an even number $\varepsilon$ using the same principle. Further, the $i$ th cycle of two local channel rectangular sequences can be expressed as

$$
\begin{aligned}
& R_{N}(n+\varepsilon N-i \varepsilon N)= u(n+\varepsilon N-i \varepsilon N) \\
&-u(n+\varepsilon N-N-i \varepsilon N), \\
& R_{N}(n+N-i \varepsilon N)=u(n+N-i \varepsilon N)-u(n-i \varepsilon N) .
\end{aligned}
$$

The original PRN code is, respectively, multiplied by the two-channel cycle rectangular sequences to structure the two new local channel sequences, which are expressed as

$$
\begin{aligned}
& H_{1}(n)=C(n+\tau) \sum_{i=1}^{M}\left((-1)^{i \varepsilon-\varepsilon} R_{N}(n+\varepsilon N-i \varepsilon N)\right), \\
& H_{2}(n)=C(n+\tau) \sum_{i=1}^{M}\left((-1)^{i \varepsilon-1} R_{N}(n+N-i \varepsilon N)\right),
\end{aligned}
$$

where $C(n+\tau)$ is the delay PRN code and $\tau$ is the time delay.
The beforehand processing received signal is executed by the correlation circumferential arithmetic with $X_{1}(n)$ and $X_{2}(n)$, respectively, which are expressed as

$$
\begin{aligned}
& X_{1}(n) \\
& =S_{\mathrm{BOC}}(n) \otimes H_{1}(n) \\
& =\left[d(n) C(n) \sum_{j=1}^{\varepsilon M}\left((-1)^{j+1} R_{N}(n+N-j N)\right) S_{\Delta}(n)+\lambda_{0}(n)\right] \\
& \otimes\left[C(n+\tau) \sum_{i=1}^{M}\left((-1)^{i \varepsilon-\varepsilon} R_{N}(n+\varepsilon N-i \varepsilon N)\right)\right] \\
& \approx \wedge_{T_{s}}(n) S_{\Delta}(n)-\wedge_{T_{s}}\left(n+T_{s}\right) S_{\Delta}(n) \\
& +\wedge_{T_{s}}\left(n+2 T_{s}\right) S_{\Delta}(n)-\cdots+\lambda_{0}(n) \\
& =\sum_{i=0}^{\varepsilon}(-1)^{\varepsilon} \wedge_{T_{s}}\left(n+i T_{s}\right) S_{\Delta}(n)+\lambda_{0}(n), \\
& X_{2}(n) \\
& =S_{\mathrm{BOC}}(n) \otimes H_{2}(n) \\
& =\left[d(n) C(n) \sum_{j=1}^{\varepsilon M}\left((-1)^{j+1} R_{N}(n+N-j N)\right) S_{\Delta}(n)+\lambda_{0}(n)\right] \\
& \otimes\left[C(n+\tau) \sum_{i=1}^{M}\left((-1)^{i \varepsilon-1} R_{N}(n+N-i \varepsilon N)\right)\right] \\
& \approx \wedge_{T_{s}}(n) S_{\Delta}(n)-\wedge_{T_{s}}\left(n-T_{s}\right) S_{\Delta}(n) \\
& +\wedge_{T_{s}}\left(n-2 T_{s}\right) S_{\Delta}(n)-\cdots+\lambda_{0}(n) \\
& =\sum_{i=0}^{\varepsilon}(-1)^{\varepsilon} \wedge_{T_{s}}\left(n-i T_{s}\right) S_{\Delta}(n)+\lambda_{0}(n) \text {, }
\end{aligned}
$$

where $\wedge_{T_{s}}(n)$ is the trigonometry sequence of width $T_{s}$ and is expressed as

$$
\wedge_{T_{s}}(n)= \begin{cases}\frac{2}{T_{s}} n+1, & -\frac{T_{s}}{2} \leq n<0 \\ -\frac{2}{T_{s}} n+1, & 0 \leq n \leq \frac{T_{s}}{2} \\ 0, & \text { other. }\end{cases}
$$

When $\varepsilon$ is five, the autocorrelation result of the BOC base-band signal is shown in Figure 5(a), and the two structured correlation results are shown in Figures 5(b) and 5(c), respectively. The results show that the positions of the two channel main peaks exactly coincide with the position of the autocorrelation main peak, and the numbers of peaks are the same in both channels. In addition, the positions of the two channel peaks are symmetrical about the main peak position of the autocorrelation function. 


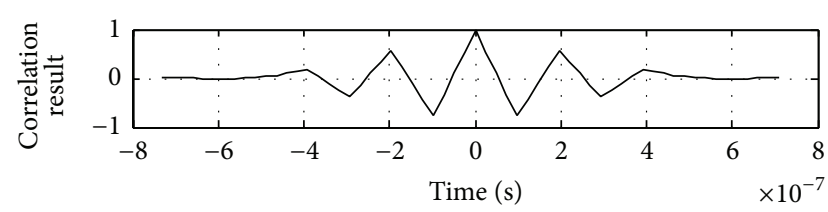

(a)

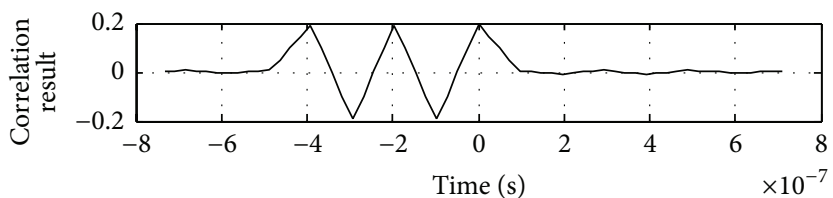

(b)

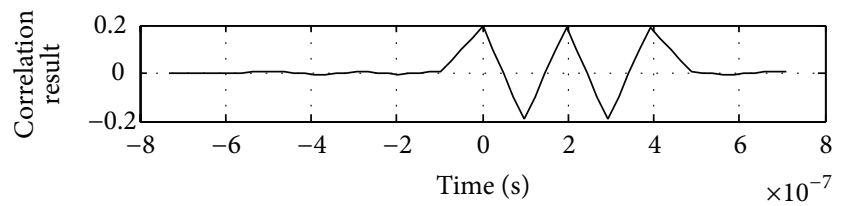

(c)

FIGURE 5: The correlation result of BOC.

In view of these characteristics and combining (8) and (9), the addition and subtraction operations are performed by using the two structured correlation results, expressed as

$$
\begin{aligned}
& \Delta X_{1}(n)=X_{1}(n)+X_{2}(n), \\
& \Delta X_{2}(n)=X_{1}(n)-X_{2}(n) .
\end{aligned}
$$

Thus, the new correlation function is structured to eliminate side peaks, and the processing is expressed as

$$
\begin{aligned}
\Delta X(n)= & \left|\Delta X_{1}(n)\right|-\left|\Delta X_{2}(n)\right| \\
\approx & \mid \sum_{i=0}^{\varepsilon}(-1)^{\varepsilon} \wedge_{T_{s}}\left(n+i T_{s}\right) S_{\Delta}(n)+\lambda_{0}(n) \\
& +\sum_{i=0}^{\varepsilon}(-1)^{\varepsilon} \wedge_{T_{s}}\left(n-i T_{s}\right) S_{\Delta}(n)+\lambda_{0}(n) \mid \\
& -\mid \sum_{i=0}^{\varepsilon}(-1)^{\varepsilon} \wedge_{T_{s}}\left(n+i T_{s}\right) S_{\Delta}(n)+\lambda_{0}(n) \\
\approx & -\sum_{i=0}^{\varepsilon}(-1)^{\varepsilon} \wedge_{T_{s}}\left(n-i T_{s}\right) S_{\Delta}(n)+\lambda_{T_{s}}(n) S_{\Delta}(n)+\lambda_{0}(n) \mid \\
& +\left|\sum_{i=1}^{\varepsilon}(-1)^{\varepsilon} \wedge_{T_{s}}\left(n+i T_{s}\right) S_{\Delta}(n)+\lambda_{0}(n)\right| \\
& +\left|\sum_{i=1}^{\varepsilon}(-1)^{\varepsilon} \wedge_{T_{s}}\left(n-i T_{s}\right) S_{\Delta}(n)+\lambda_{0}(n)\right|
\end{aligned}
$$

$$
\begin{aligned}
& -\left|\sum_{i=1}^{\varepsilon}(-1)^{\varepsilon} \wedge_{T_{s}}\left(n+i T_{s}\right) S_{\Delta}(n)+\lambda_{0}(n)\right| \\
& -\left|\sum_{i=1}^{\varepsilon}(-1)^{\varepsilon} \wedge_{T_{s}}\left(n-i T_{s}\right) S_{\Delta}(n)+\lambda_{0}(n)\right| \\
& \approx 2\left|\wedge_{T_{s}}(n) S_{\Delta}(n)\right|+\left|\lambda_{0}(n)\right| .
\end{aligned}
$$

When the impacts of the frequency error and noise function are likely to be relatively weak, the relationship of the main peak value $A_{1}$ in the $\Delta X(n)$ function and the BOC autocorrelation function value $A_{2}$ is expressed as

$$
A_{1}=\frac{2}{\varepsilon} A_{2} .
$$

To improve the peak, the result of $\Delta X(n)$ is multiplied by a coefficient of $\varepsilon / 2$ to obtain the final expression as

$$
\Delta X^{\prime}(n)=\frac{\varepsilon}{2} \cdot \Delta X(n)=\varepsilon\left|\wedge_{T_{s}}(n) S_{\Delta}(n)\right|+\frac{\varepsilon}{2}\left|\lambda_{0}(n)\right| .
$$

\section{Performance Analysis}

The $\Delta X_{1}(n)$ and $\Delta X_{2}(n)$ may be approximately represented by

$$
\begin{aligned}
& \Delta X_{1}(n) \\
& =S_{\mathrm{BOC}}(n) \otimes H_{1}(n)+S_{\mathrm{BOC}}(n) \otimes H_{2}(n) \\
& =S_{\mathrm{BOC}}(n) \otimes\{C(n+\tau) \\
& \quad \cdot\left[\sum_{i=1}^{M}\left((-1)^{i \varepsilon-\varepsilon} R_{N}(n+\varepsilon N-i \varepsilon N)\right)\right. \\
& \left.\left.\quad+\sum_{i=1}^{M}\left((-1)^{i \varepsilon-1} R_{N}(n+N-i \varepsilon N)\right)\right]\right\},
\end{aligned}
$$

$$
\begin{aligned}
\Delta & X_{2}(n) \\
= & S_{\mathrm{BOC}}(n) \otimes H_{1}(n)-S_{\mathrm{BOC}}(n) \otimes H_{2}(n) \\
= & S_{\mathrm{BOC}}(n) \otimes\{C(n+\tau) \\
& \cdot\left[\sum_{i=1}^{M}\left((-1)^{i \varepsilon-\varepsilon} R_{N}(n+\varepsilon N-i \varepsilon N)\right)\right. \\
& \left.\left.\quad-\sum_{i=1}^{M}\left((-1)^{i \varepsilon-1} R_{N}(n+N-i \varepsilon N)\right)\right]\right\} .
\end{aligned}
$$


At the same time, the structured square function can be expressed as

$$
\begin{aligned}
& \sum_{i=1}^{M}\left((-1)^{i \varepsilon-\varepsilon} R_{N}(n+\varepsilon N-i \varepsilon N)\right) \\
& +\sum_{i=1}^{M}\left((-1)^{i \varepsilon-1} R_{N}(n+N-i \varepsilon N)\right)=\frac{\varepsilon N}{2}, \\
& \sum_{i=1}^{M}\left((-1)^{i \varepsilon-\varepsilon} R_{N}(n+\varepsilon N-i \varepsilon N)\right) \\
& -\sum_{i=1}^{M}\left((-1)^{i \varepsilon-1} R_{N}(n+N-i \varepsilon N)\right)=0 .
\end{aligned}
$$

Hence, $\Delta X_{1}(n)$ satisfies a Gaussian distribution whose mean is $(A / 4) \varepsilon N$ and whose variance is $\sigma^{2} \varepsilon N / 2$, and $\Delta X_{2}(n)$ satisfies Gaussian distribution whose mean is 0 and whose variance is $\sigma^{2} \varepsilon N / 2$.

Where $A$ is the signal amplitude and $\sigma^{2}$ is the noise variance, the probability density function $\left|\Delta X_{1}(n)\right|$ is expressed as

$$
f_{1}(x)=\frac{1}{\sqrt{\pi \sigma^{2} \varepsilon N}}\left(e^{-(x-(A / 4) \varepsilon N)^{2} / \sigma^{2} \varepsilon N}+e^{-(x+(A / 4) \varepsilon N)^{2} / \sigma^{2} \varepsilon N}\right)
$$

and the probability density function $\left|\Delta X_{2}(n)\right|$ is expressed as

$$
f_{2}(x)=\frac{2}{\sqrt{\pi \sigma^{2} \varepsilon N}} e^{-(x)^{2} / \sigma^{2} \varepsilon N} .
$$
as

Thus, the $\Delta X(n)$ probability density function is expressed

$$
\begin{aligned}
f(x)= & \frac{1}{\sqrt{4 \pi \sigma^{2} \varepsilon N}} \\
& \cdot\left(e^{-(x-(A / 4) \varepsilon N)^{2} / 2 \sigma^{2} \varepsilon N}+e^{-(x+(A / 4) \varepsilon N)^{2} / 2 \sigma^{2} \varepsilon N}\right) .
\end{aligned}
$$

The false alarm probability of the ISPA algorithm is expressed as

$$
P_{f a}=\int_{G}^{+00} \frac{1}{\sqrt{2 \pi \varepsilon N} \sigma} e^{-x^{2} / 2 \varepsilon N \sigma^{2}} d x
$$

The acquisition detection probability of the ISPA algorithm is expressed as

$$
\begin{aligned}
P_{D}= & \int_{G}^{+00} \frac{1}{\sqrt{4 \pi \sigma^{2} \varepsilon N}} \\
& \cdot\left(e^{-(x-(A / 4) \varepsilon N)^{2} / 2 \sigma^{2} \varepsilon N}+e^{-(x+(A / 4) \varepsilon N)^{2} / 2 \sigma^{2} \varepsilon N}\right) d x
\end{aligned}
$$

where $G$ is the acquisition threshold.

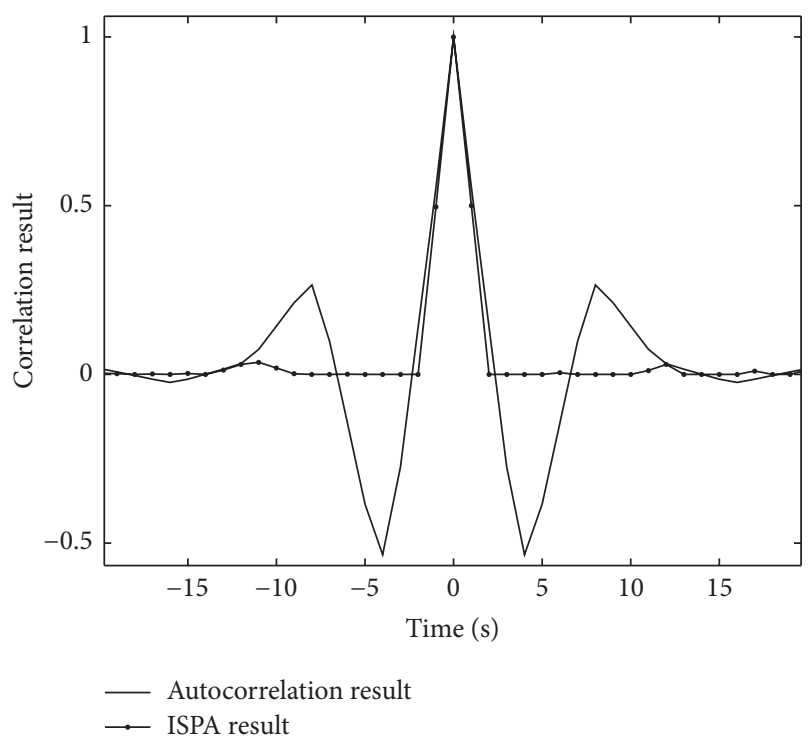

FIGURE 6: The ISPA result for $\sin B O C(15,10)$.

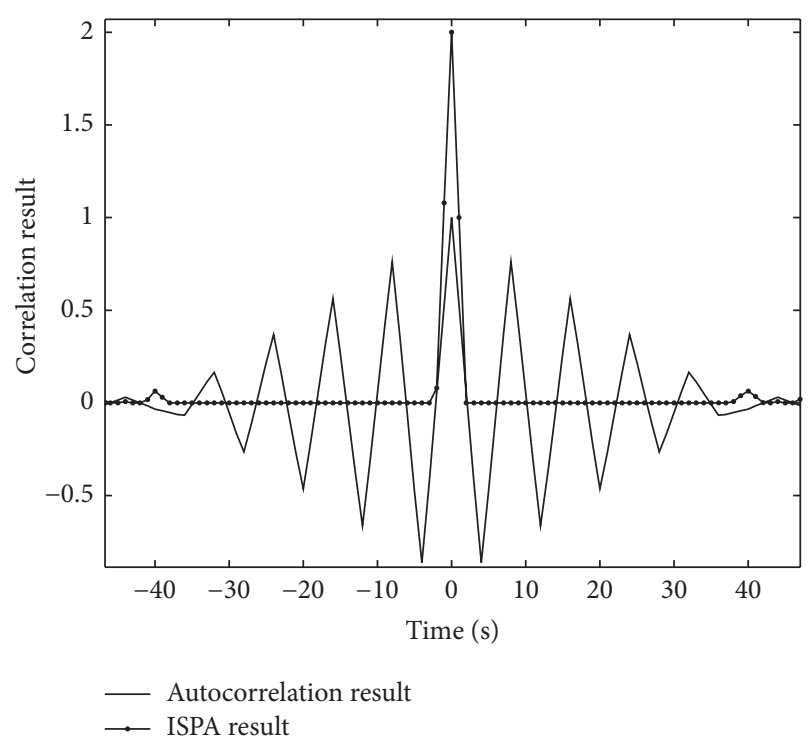

Figure 7: The ISPA result for $\sin B O C(10,2)$.

\section{Analysis and Simulation}

5.1. Side Peak Inhibition Analysis. Equations (9) and (13) show that the final correlation result has a single peak whose main waveform is a triangular peak. Thus, the ISPA algorithm can achieve the goal of side peak inhibition. The new algorithm is then simulated using the following parameters: $10.23 \mathrm{MHz}$ PRN code frequency, $15.345 \mathrm{MHz}$ square wave frequency, and 122.76 MHz sampling frequency, modulation order of 3 , and the sine-phased BOC signal for these parameters is expressed as $\sin B O C(15,10)$.

The ISPA result for $\sin \operatorname{BOC}(15,10)$ is shown in Figure 6 . The ISPA results for $\sin \operatorname{BOC}(10,2), \operatorname{cosBOC}(10,5)$, and $\cos \mathrm{BOC}(6,1)$ are shown in Figures 7,8 , and 9, respectively. The simulation results show that the ISPA algorithm can 


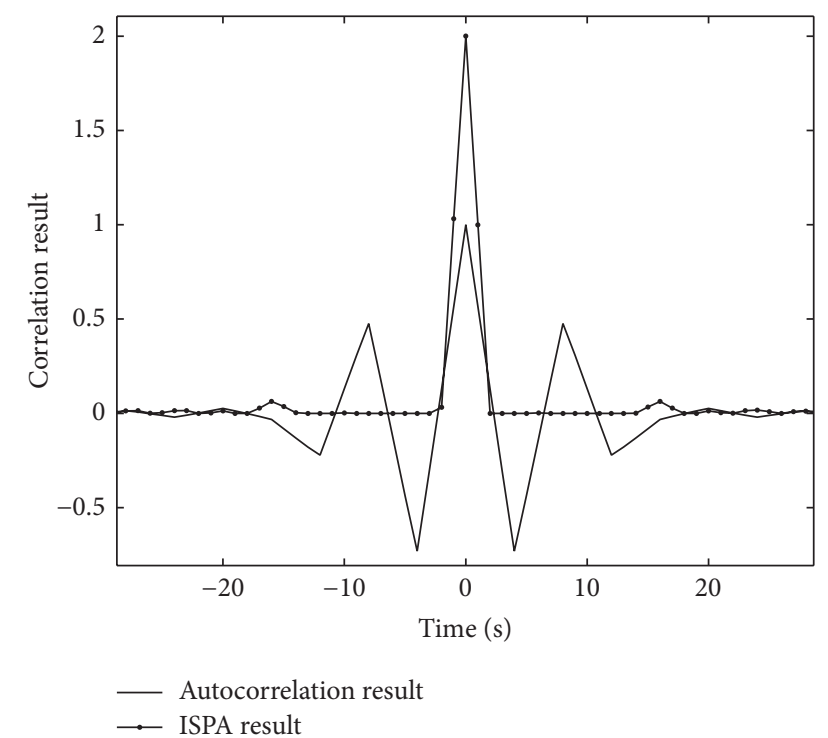

FIGURE 8: The ISPA result for $\cos B O C(10,5)$.

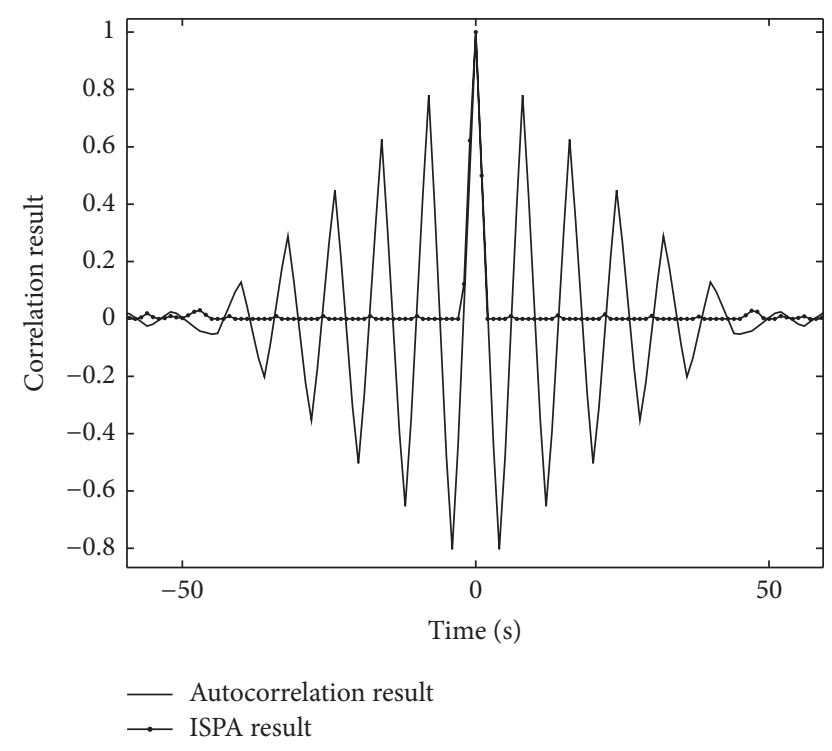

Figure 9: The ISPA result for $\cos B O C(6,1)$.

clearly recover the main peak, whose position is the same as the main peak position of the autocorrelation function. In particular, the ISPA algorithm can effectively inhibit side peaks.

5.2. Adaptability Analysis. The new algorithm result is influenced by the frequency error and the mixed noise, according to (13). The algorithm result approximately conforms to the cycle equation because of the frequency error function cycle characteristics. When the relationship of the frequency error and accumulation time $t$ satisfies (21), the algorithm result error reaches its maximum. We also find that the ISPA

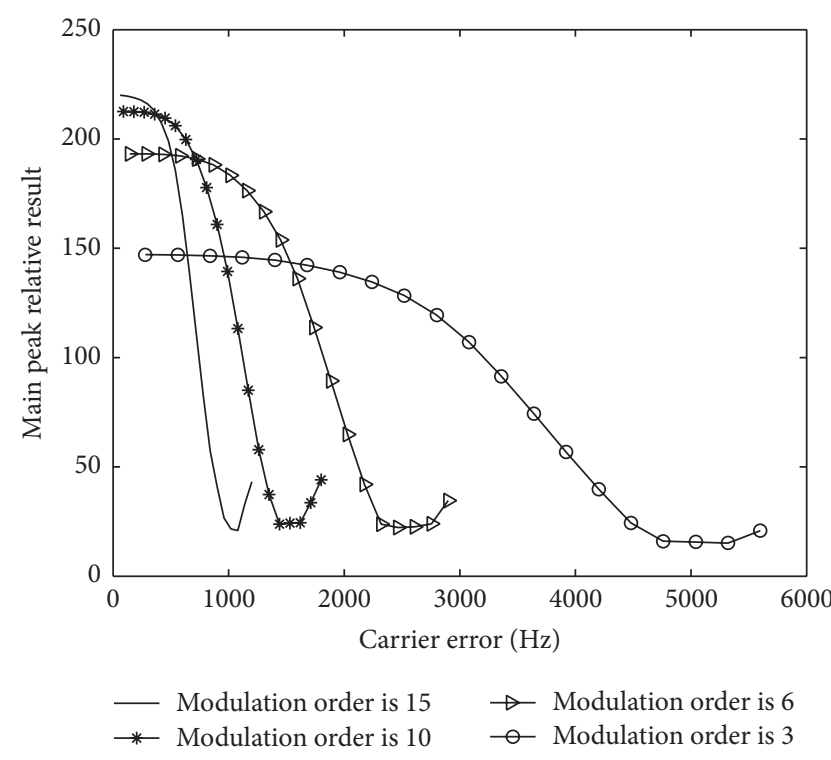

FIGURE 10: The relationship between the relative main peak and frequency error.

algorithm result decreases gradually along with the increase of mixed noise, according to

$$
S_{\Delta}(n)=\frac{k}{2 t},
$$

where $k$ is the positive integer.

Furthermore, the ISPA algorithm's adaptability is simulated with the following parameters: $15.345 \mathrm{MHz}$ square wave frequency and $122.76 \mathrm{MHz}$ sampling frequency, modulation mode is sine mode, and modulation orders are 15, 10, 6, and 3, respectively.

The relationship between the relative main peak and frequency error is shown in Figure 10, which shows that the results satisfy (21). The relationship between the relative main peak and SNR is shown in Figure 11, revealing that the relative main peak decreases gradually with decreasing SNR. And the ISPA algorithm's adaptability to the SNR environment is more than $-25 \mathrm{~dB}$, according to (13) and Figure 11.

5.3. Superiority Analysis. To verify the superiority of the ISPA algorithm, this ISPA algorithm is compared with other algorithms, namely, the FBA algorithm, POA algorithm, and SPRA algorithm. The simulation parameters are as follows: 2.046 MHz PRN code frequency, and the modulation mode is sine mode.

With changing modulation order, the main peak width changes and the main peak relative changes are shown in Figures 12 and 13. The results show that the ISPA algorithm's main peak width is the smallest, and its main peak relative result is the greatest, demonstrating that this algorithm's acquisition and tracking performance is the best.

The side peak relative changes and the main/side peak ratio changes with changing modulation order are shown in Figures 14 and 15. The results show that the ISPA algorithm side peak relative result is the smallest, and the main/side 


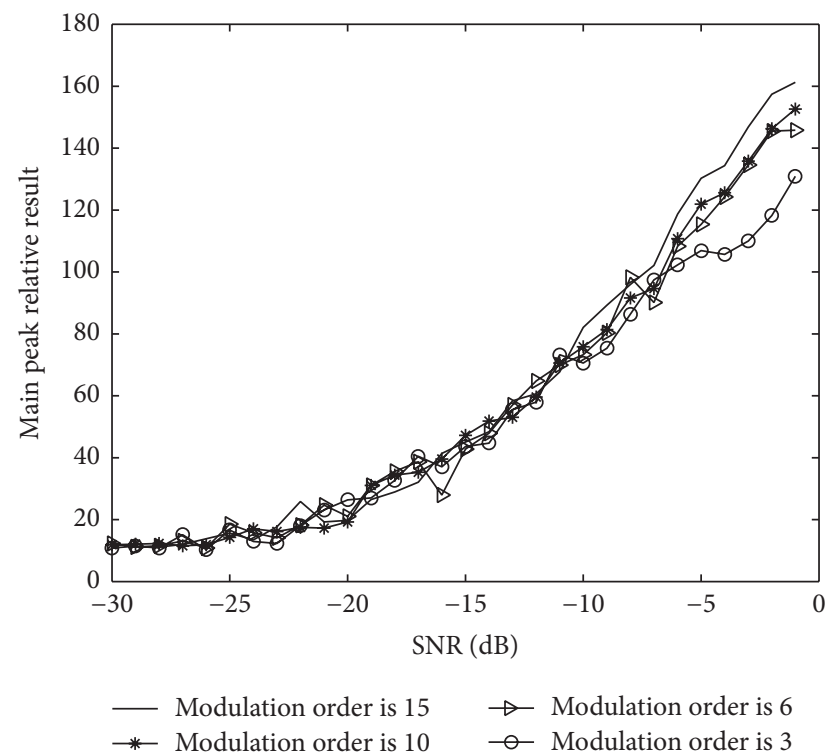

FIGURE 11: The relationship between the relative main peak and SNR.

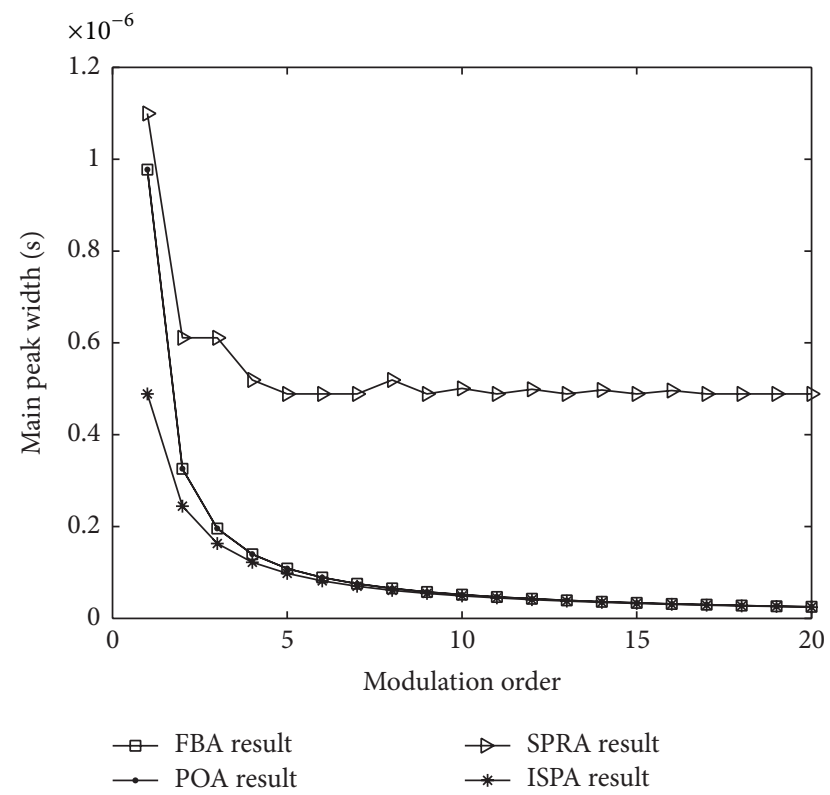

FIGURE 12: The relationship between the main peak width and the modulation order.

peak ratio is the greatest, demonstrating that this algorithm's side peak inhibition ability is best.

The main peak relative changes with changing SNR are shown in Figure 16. The results show that the adaptability of the ISPA algorithm is better than the FBA algorithm and POA algorithm, but there are no significant differences between the ISPA algorithm and the SPRA algorithm.

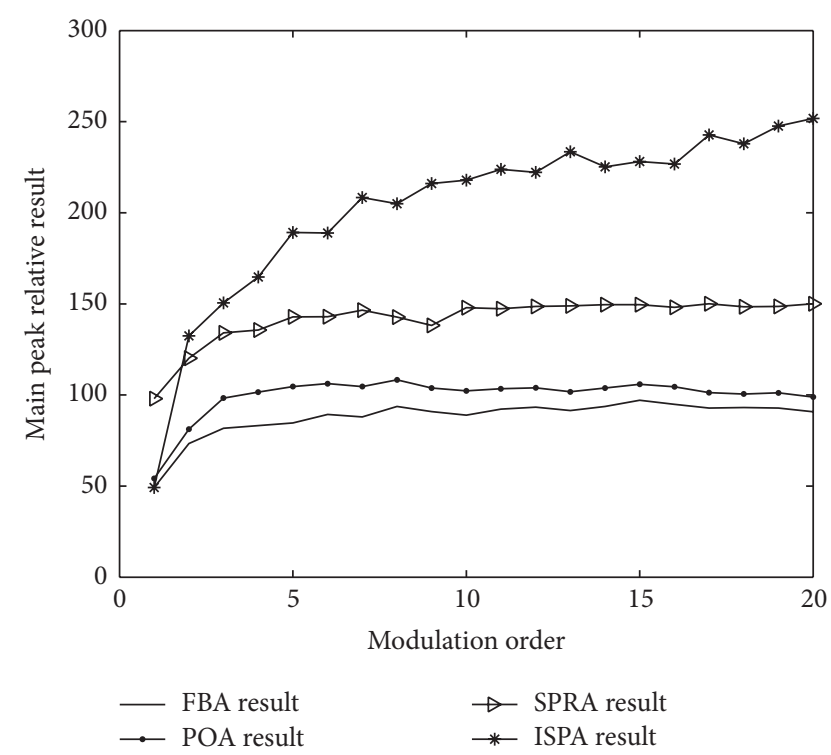

FIGURE 13: The relationship between the relative main peak and the modulation order.

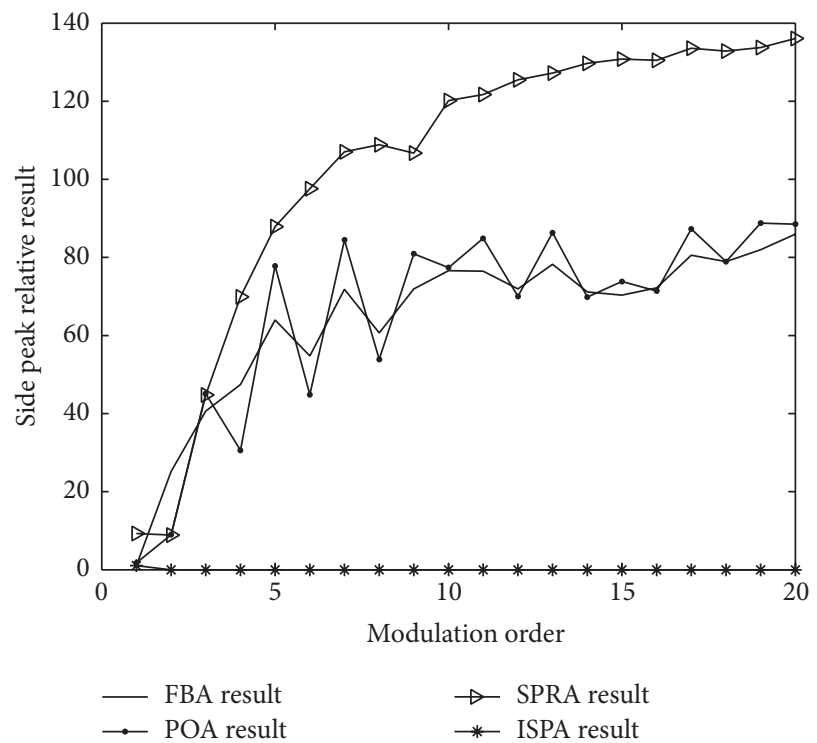

FIGURE 14: The relationship between the relative side peak and the modulation order.

\section{Conclusions}

In this paper, the principle and characteristics of BOC modulation signals have been studied. To implement the BOC modulated signal acquisition, effective algorithms have been studied, including the full band acquisition (FBA) algorithm, the peak optimization acquisition (POA) algorithm, and the single peak recovery acquisition (SPRA) algorithm. Considering the filter restriction and generic deficiency problems in traditional algorithms, we propose the ISPA algorithm. We eliminate all side peaks of the BOC correlation function (CF) by structuring special sequences composed of PRN code and cycle rectangular sequences. The ISPA algorithm can be 


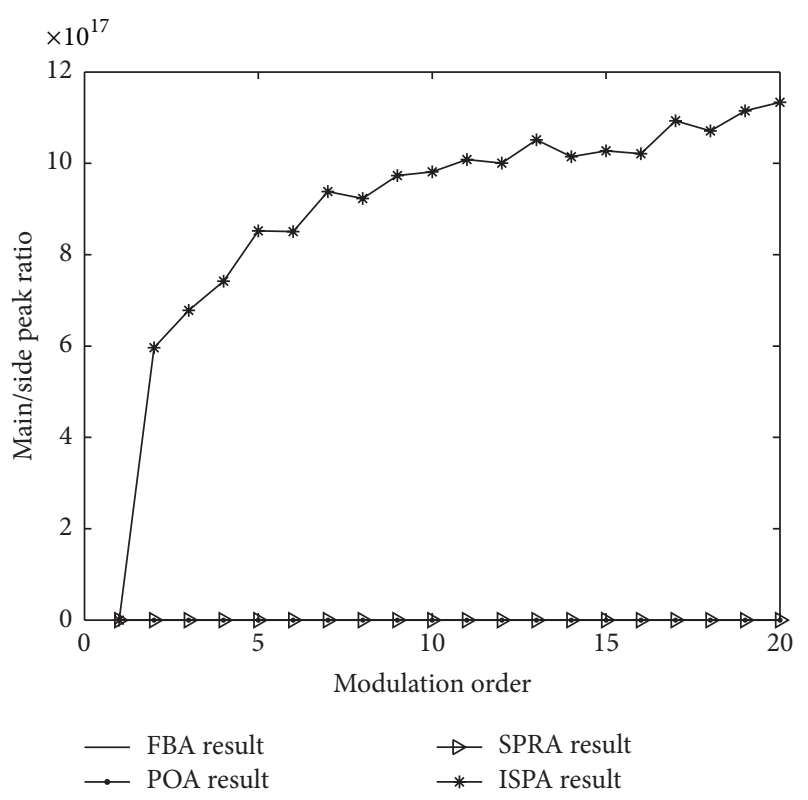

FIGURE 15: The relationship between the main/side peak ratio and the modulation order.

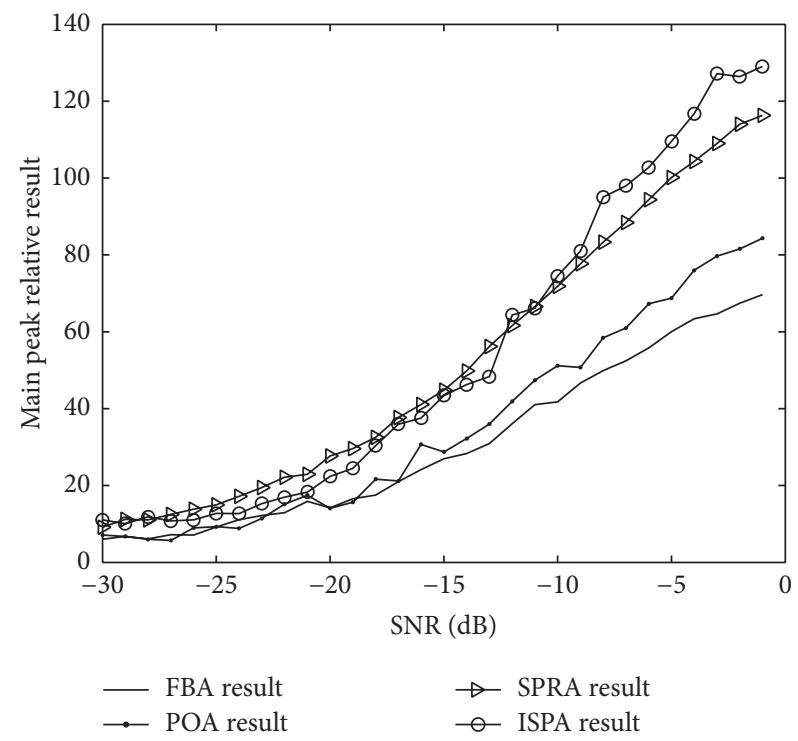

FIGURE 16: The relationship between the relative main peak changes and SNR.

applied to both generic sine- and cosine-phased BOC signals and to all modulation orders. In addition, it outperforms the traditional algorithms in acquisition, inhibition side peak ability, and adaptability to lower SNR.

\section{Conflict of Interests}

The authors declare that there is no conflict of interests regarding the publication of this paper.

\section{Acknowledgments}

This work was supported by the Program for Liaoning Innovative Research Team in University (no. LT2011005), New Century Program for Excellent Talents of Ministry of Education of China (no. NCET-11-1013), Project of Science and Technology Department of Liaoning Province (no. 20121038), Project of Education Department of Liaoning Province (no. L2013085), and the Open Foundation of Key Laboratory of Shenyang Ligong University.

\section{References}

[1] K. Subburaj, S. Bhatara, J. Tangudu, J. R. Samuel, R. Ganesan, and K. Ramasubramanian, "Spur mitigation in high-sensitivity GNSS receivers," IEEE Transactions on Circuits and Systems II: Express Briefs, vol. 61, no. 2, pp. 100-104, 2014.

[2] R. R. Rick and L. B. Milstein, "Optimal decision strategies for acquisition of spread-spectrum signals in frequency-selective fading channels," IEEE Transactions on Communications, vol. 46, no. 5, pp. 686-694, 1998.

[3] X. Li and W. Guo, "Efficient differential coherent accumulation algorithm for weak GPS signal bit synchronization," IEEE Communications Letters, vol. 17, no. 5, pp. 936-939, 2013.

[4] T. H. Ta, N. C. Shivaramaiah, A. G. Dempster, and L. L. Presti, "Significance of cell-correlation phenomenon in GNSS matched filter acquisition engines," IEEE Transactions on Aerospace and Electronic Systems, vol. 48, no. 2, pp. 1264-1286, 2012.

[5] P. Fishman and J. W. Betz, "Predicting performance of direct acquisition for the M-code signal," in Proceedings of the International Technical Meeting of the Institute of Navigation (IONNTM '00), pp. 574-582, 2000.

[6] J. Betz and P. Capozza, "System for direct acquisition of received signals," US patent no. 2004/0071200 A1, 2004.

[7] N. Martin, V. Leblond, G. Guillotel, and V. Heiries, "BOC(x,y) signal acquisition techniques and performances," in Proceedings of the 16th International Technical Meeting of the Satellite Division of the Institute of Navigation (ION GPS/GNSS '03), pp. 188-198, 2003.

[8] A. Burian, E. S. Lohan, and M. Renfors, "BPSK-like methods for hybrid-search acquisition of galileo signals," in Proceedings of the IEEE International Conference on Communications (ICC '06), pp. 5211-5216, July 2006.

[9] W.-L. Mao, C.-S. Hwang, C.-W. Hung, J. Sheen, and P.-H. Chen, "Unambiguous BPSK-like CSC method for Galileo acquisition," in Proceedings of the 18th International Conference on Methods and Models in Automation and Robotics (MMAR '13), pp. 627632, Międzyzdroje, Poland, August 2013.

[10] B. Kim and S.-H. Kong, "Two-dimensional compressed correlator for fast acquisition of BOC $(\mathrm{m}, \mathrm{n})$ signals," IEEE Transactions on Vehicular Technology, vol. 63, no. 6, pp. 2662-2672, 2014.

[11] F. Benedetto, G. Giunta, E. S. Lohan, and M. Renfors, "A fast unambiguous acquisition algorithm for BOC-modulated signals," IEEE Transactions on Vehicular Technology, vol. 62, no. 3, pp. 1350-1355, 2013.

[12] Z. Yao, M. Lu, and Z. Feng, "Unambiguous sine-phased binary offset carrier modulated signal acquisition technique," IEEE Transactions on Wireless Communications, vol. 9, no. 2, pp. 577580, 2010.

[13] O. Julien, C. Macabiau, M. E. Cannon, and G. Lachapelle, "ASPeCT: unambiguous sine-BOC $(\mathrm{n}, \mathrm{n})$ acquisition/tracking 
technique for navigation applications," IEEE Transactions on Aerospace and Electronic Systems, vol. 43, no. 1, pp. 150-162, 2007.

[14] Z. Yao, X. Cui, M. Lu, Z. Feng, and J. Yang, "Pseudo-correlationfunction-based unambiguous tracking technique for sine-BOC signals," IEEE Transactions on Aerospace and Electronic Systems, vol. 46, no. 4, pp. 1782-1796, 2010.

[15] Y. Zhou, X. Hu, T. Ke, and Z. Tang, "Ambiguity mitigating technique for cosine-phased binary offset carrier signal," IEEE Transactions on Wireless Communications, vol. 11, no. 6, pp. 1981-1984, 2012.

[16] S. Fischer, A. Guérin, and S. Berberich, "Acquisition concepts for galileo BOC $(2,2)$ signals in consideration of hardware limitations," in Proceedings of the IEEE 59th Vehicular Technology Conference (VTC-Spring '04), pp. 2852-2856, May 2004.

[17] Y. Feng, M. Xu, X. Liu, and F. Liu, "Main lobe overlapped acquisition algorithm of frequency domain based on BOC modulation signal," Journal of Data Acquisition \& Processing, vol. 27, no. 1, pp. 27-31, 2012.

[18] L. Yang, Y. Feng, C. Pan, and Y. Bo, "The research of side-band acquisition for BOC-modulated signal," in Proceedings of the International Conference on Wireless Communications, Networking and Mobile Computing, pp. 645-648, September 2007. 


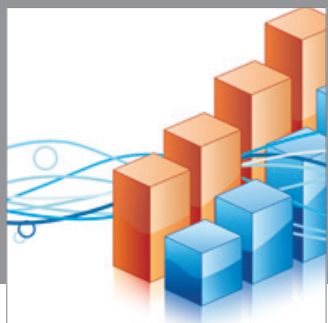

Advances in

Operations Research

mansans

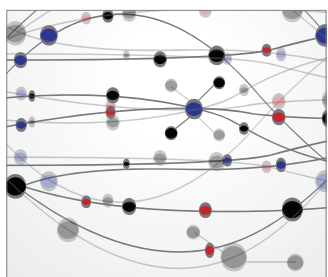

The Scientific World Journal
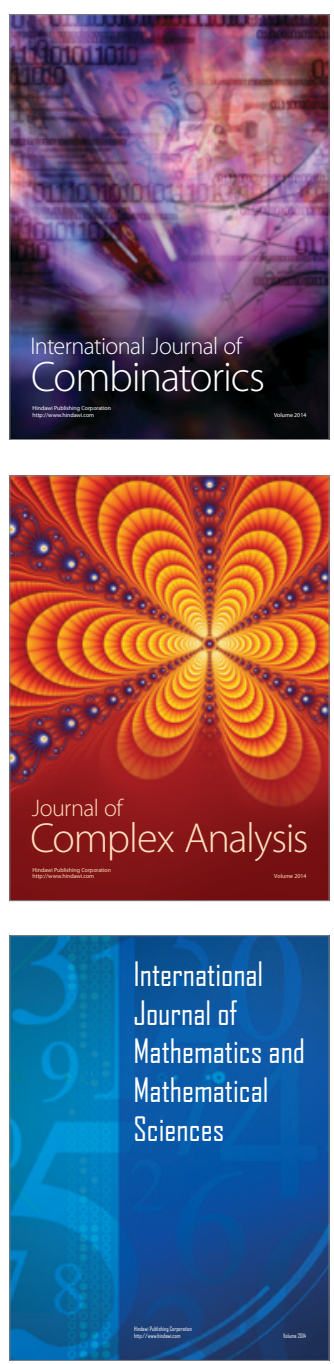
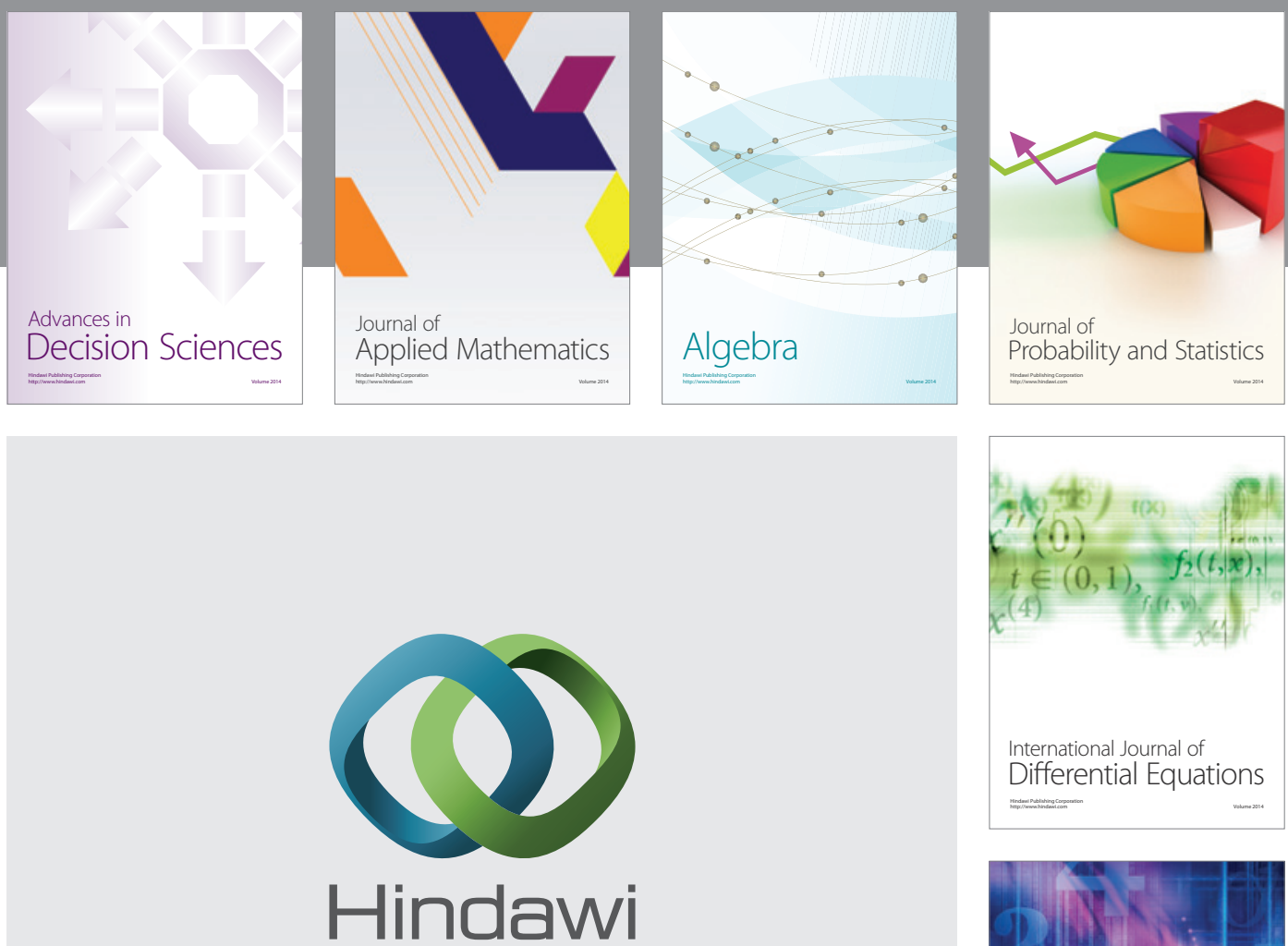

Submit your manuscripts at http://www.hindawi.com
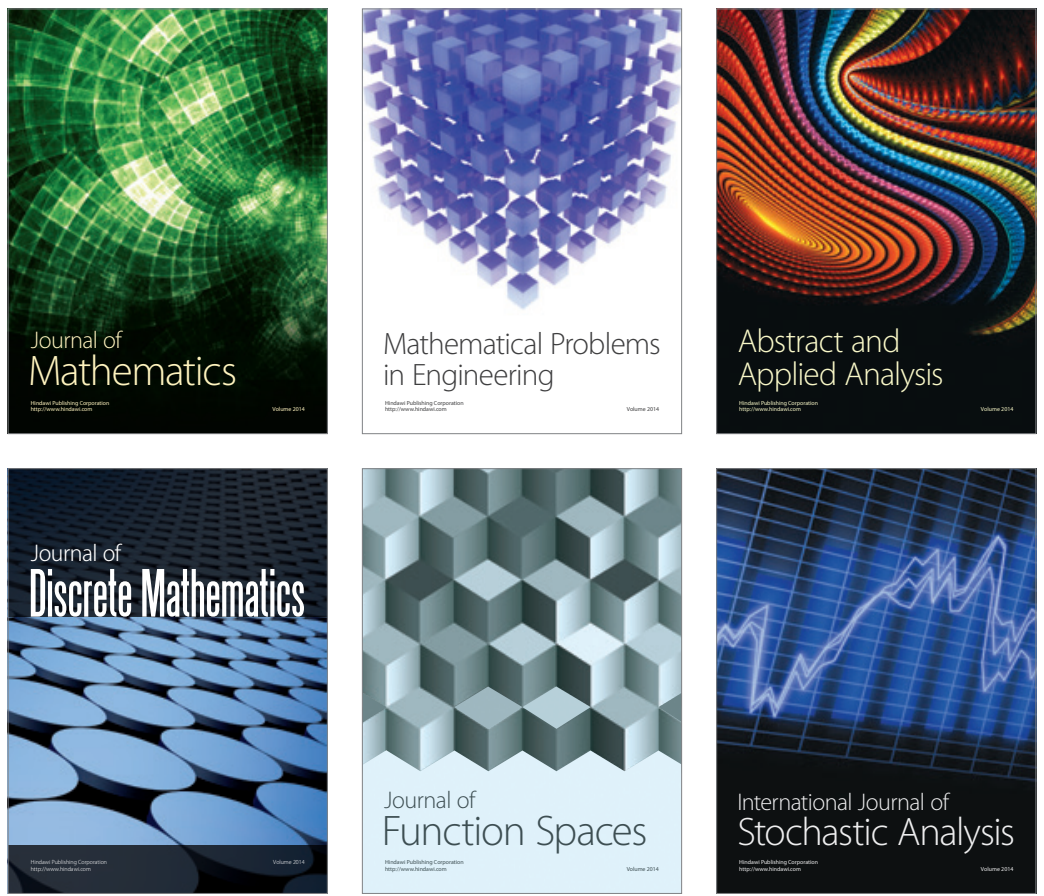

Journal of

Function Spaces

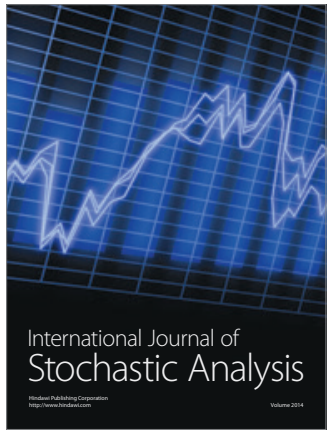

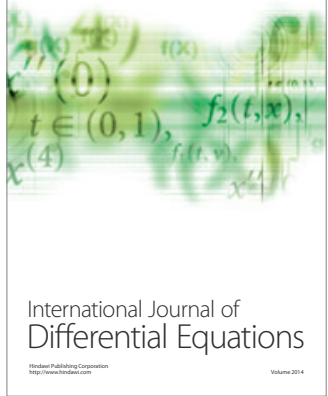
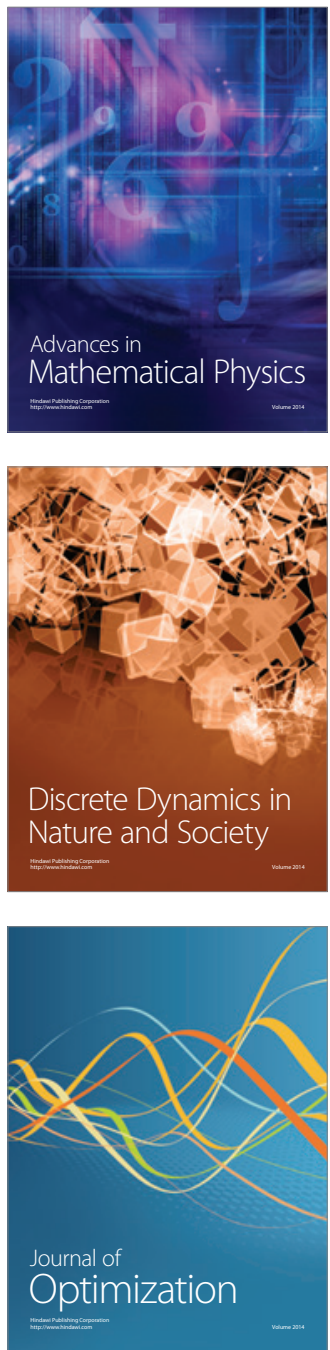